9 Ricard JD, Messika J. Low-dose corticosteroids during severe community-acquired pneumonia: end of the story. Eur Respir J 2015; 45: 305-307.

\title{
Computed tomography in the diagnosis of bronchiectasis
}

To the Editor:

We read with great interest the article by HuRsT et al. [1], who discussed the overlap between chronic obstructive pulmonary disease (COPD) and bronchiectasis, and provided the position statement from the BRONCH-UK Consortium.

Bronchiectasis is defined as abnormal, persistent bronchial dilatation, usually associated with inflammation in the bronchial tree and lung parenchyma. The disease remains a common cause of significant morbidity and mortality, especially when associated with COPD. Computed tomography (CT) is now the diagnostic modality of choice and may also contribute to clinical management. However, we would like to highlight some technical aspects of diagnosis.

Bronchial dilatation remains the most important CT finding to establish a diagnosis of bronchiectasis, and its detection typically relies solely on simple visual identification [2]. Although generally accurate, visual inspection may lead to the overestimation of airway dilatation, as a result of a subtle optical illusion in which the diameters of hollow circles appear larger than those of solid circles, despite their identical size, a phenomenon that only occurs when airways are sectioned at right angles. Several quantitative metrics, most including lumen diameter and broncho-arterial ratio (ring sign), have been proposed for the diagnosis of this condition. Care should be taken to avoid misinterpretation of physiologically dilated airways as bronchiectatic, which occurs, for example, in individuals who live at high altitudes [3], in elderly patients [2], or on images obtained through the lung bases in the prone position. In one study of high-resolution CT (HRCT) findings in 85 subjects without cardiopulmonary disease divided into three age groups, a significant correlation between the broncho-arterial ratio and age was noted $(\mathrm{r}=0.768$, $\mathrm{p}<0.0001$ ), with broncho-arterial ratios $>1$ identified in $41 \%$ of individuals aged $>65$ years [2]. With ageing, morphological changes occur in the lung, which include: increased alveolar duct air; decreased complexity of the alveolar surface or surface-to-volume ratio; loss of alveolar wall tissue, elastic tissue, and bronchiolar muscle; and increased frequency of emphysema [4]. The relationships between ageing and morphological changes of the lung have also been investigated radiographically. LeE et al. [5] detected age-related increases in the frequency and severity of air trapping on HRCT images of the lung. Air trapping is induced by occlusion or narrowing of the airway. LEE et al. [5] suggested that age-related occlusion or luminal narrowing occurred at the lobular bronchiole level. Hansell et al. [6] showed a clear relationship between the extent of bronchiectasis and CT attenuation values, which suggests that involvement of the small airways is an integral part of bronchiectasis. Although the relationship between small airways involvement and the development of bronchodilation has not yet been clearly established, these reports provide some support for a relationship between bronchodilation and age. That relationship might be another reason for the increase in the broncho-arterial ratio that occurs with age.

These important technical parameters and physiological variations should be evaluated critically, as they may dramatically influence the diagnosis and determination of the significance of bronchiectasis.

$@$ ERSpublications

Bronchiectasis diagnosis and significance can be influenced by technical parameters and physiological variations http://ow.ly/OatKb

Ricardo Holderbaum Do Amaral ${ }^{1}$, Carlos Schuler Nin ${ }^{1}$, Vinicius Valerio de Souza ${ }^{1}$, Edson Marchiori ${ }^{2}$ and Bruno Hochhegger ${ }^{1}$

${ }^{1}$ Radiology Dept, Santa Casa de Misericordia de Porto Alegre, Porto Alegre, Brazil. ${ }^{2}$ Radiology Dept, Fluminense Federal University, Porto Alegre, Brazil.

Correspondence: Ricardo Holderbaum Do Amaral, Radiology Department, Santa Casa de Misericordia de Porto Alegre, Porto Alegre, Brazil. E-mail: rh.doamaral@gmail.com 
Received: Feb 252015 | Accepted: March 172015

Conflict of interest: None declared.

\section{References}

1 Hurst JR, Elborn JS, Soyza AD, et al. COPD-bronchiectasis overlap syndrome. Eur Respir J 2015; 45: 310-313.

2 Matsuoka S, Uchiyama K, Shima $\mathrm{H}$, et al. Bronchoarterial ratio and bronchial wall thickness on high-resolution CT in asymptomatic subjects: correlation with age and smoking. AJR Am J Roentgenol 2003; 180: 513-518.

3 Lynch DA, Newell JD, Tschomper BA, et al. Uncomplicated asthma in adults: comparison of CT appearance of the lungs in asthmatic and healthy subjects. Radiology 1993; 188: 829-833.

4 Kang EY, Miller RR, Müller NL. Bronchiectasis: comparison of preoperative thin-section CT and pathologic findings in resected specimens. Radiology 1995; 195: 649-654.

5 Lee KW, Chung SY, Yang I, et al. Correlation of aging and smoking with air trapping at thin-section CT of the lung in asymptomatic subjects. Radiology 2000; 214: 831-836.

6 Hansell DM, Wells AU, Rubens MB, et al. Bronchiectasis: functional significance of areas of decreased attenuation at expiratory CT. Radiology 1994; 193: 369-374.

Eur Respir J 2015; 46: 576-577 | DOI: 10.1183/09031936.00031415 | Copyright @eERS 2015 